\title{
Rhinocerebral Mucormycosis: an Unusual Presentation
}

\author{
Rajeshwari A, Gangadhara Somayaji K. S* \\ Department of ENT, Yenepoya Medical College, Mangalore, 575003, India
}

\begin{abstract}
Rhinocerebral mucormycosis is one of the most rapidly progressing and lethal form of fungal infection in humans which usually begins in the nose and paranasal sinuses and can extend to CNS. A 68 year old lady with poorly controlled diabetes presented with fever, disorientation, headache and left sided LMN type of facial palsy. ENT examination revealed a palatal ulcer and mucopus in middle meatus. MRI revealed an infarct in the territory of left superior cerebellar artery and haziness and mucosal thickening in the left maxillary and ethmoidal sinuses. Patient underwent endoscopic medial maxillectomy and debridement of palatal ulcer. Postoperative histopathology was suggestive of zygomycosis. Patient was subsequently treated with Amphotericin B for 4 weeks with good recovery. This case is being reported for its rarity as mucormycosis presenting with cerebellar signs has not been reported in the English literature so far.
\end{abstract}

Keywords Rhinocerebral Mucormycosis, Cerebellar Involvement, Facial Palsy

\section{Introduction}

Mucormycosis is a fatal opportunistic infection caused by the fungi of the order mucorales which includes mucor, rhizopus, absidia and others. ${ }^{1}$ These organisms are ubiquitous and are found in soil and decaying organic matter. Rhizopus oryzae is the predominant pathogen accounting for $60 \%$ of all the forms of mucormycosis and $90 \%$ of rhinocerebral cases. This disease usually develops in patients who are metabolically or immunologically compromised; but at times can be seen in otherwise healthy individuals. ${ }^{2}$ Mucormycosis can manifest as one of six different clinical syndromes; rhinocerebral, pulmonary, cutaneous, gastrointestinal, central nervous system and disseminated. Rhinocerebral type is the most common and is subdivided into rhinomaxillary, rhinoorbital and rhinoorbitocerebral. ${ }^{3}$ The exact pathogenesis of rhinocerebral mucormycosis and its pathways of spread are not clearly known. It is believed that the fungus initially inoculates the nasal mucosa spreading to the paranasal sinuses, orbit and the intracranial fossa. ${ }^{4}$ Among the intracranial structures, involvement of cavernous sinus and internal carotid artery is well known. We are reporting a case of mucormycosis, presented with cerebellar signs, which has not been reported in the English literature so far. Surprisingly, orbit was spared in our case.

\section{Case report}

A 68 year old lady with poorly controlled diabetes was

* Corresponding author:

ksgsomayaji@yahoo.co.in (Gangadhara Somayaji K.S)

Published online at http://journal.sapub.org/ajmms

Copyright (C) 2012 Scientific \& Academic Publishing. All Rights Reserved admitted to the intensive care unit in a disoriented and confused state with history of fever of 15 days, unsteadiness of gait of 12 days with a tendency to fall towards the left side and headache of 7 days duration. There was also a history of swelling and pain over the left side of the face and deviation of the angle of the mouth to right side of 2 days duration. (Fig No.1)

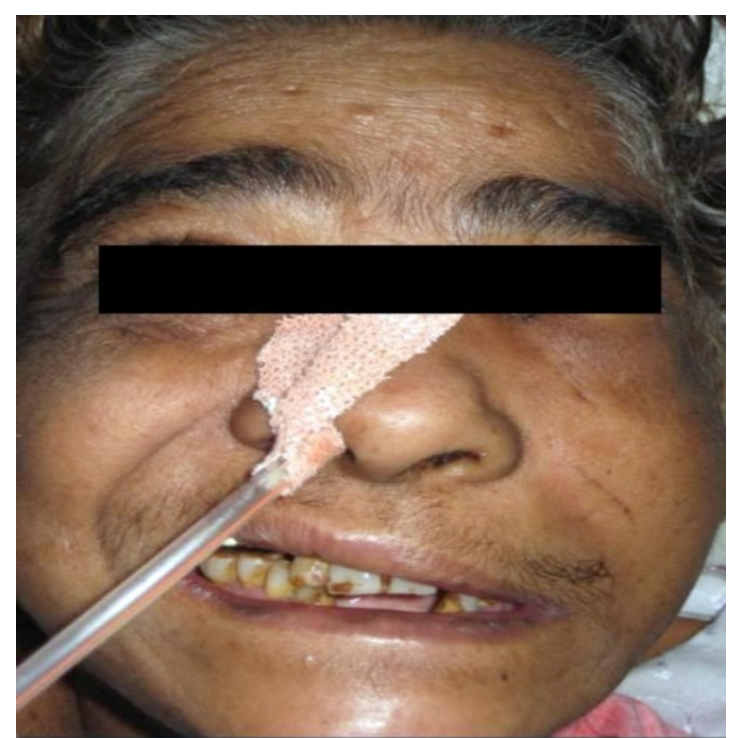

Figure 1. Showing left sided facial swelling and LMN Facial palsy

On examination, patient was disoriented and had unsteadiness of gait with tendency to fall towards the left side. There was a left sided lower motor neuron type of grade 4 facial palsy and signs of cerebellar dysfunction. On ENT examination, there was a tender diffuse indurated swelling over the left maxilla with mucopus seen in the left middle meatus. Oral cavity examination revealed a white necrotic patch on the hard palate on the left side. (Fig.No.2) Rest of 
the ENT, Opthalmology and neurology examination was normal.

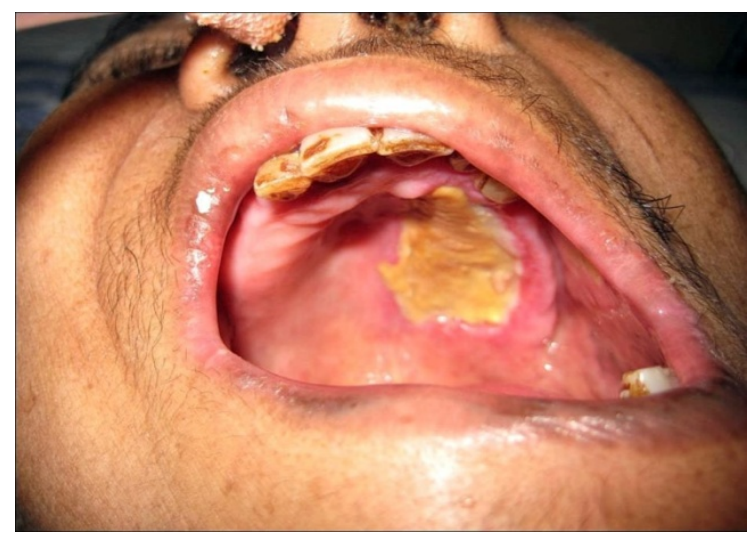

Figure 2. Showing necrotic patch over the hard palate

Routine blood investigations were normal except for elevated ESR. ( $80 \mathrm{~mm}$ of $\mathrm{Hg}$ after one hour) and high fasting blood sugar. (400 mg/dl) MRI of the brain with contrast revealed an acute infarct of left superior cerebellar artery territory involving the superior aspect of left cerebellar hemisphere. (Fig.No.3)

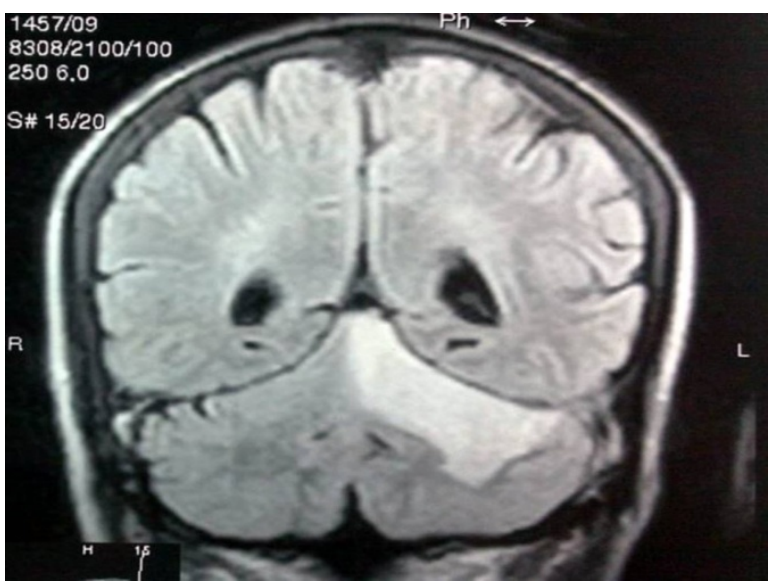

Figure 3. Showing infarct in left cerebellar hemisphere

MRI of the PNS showed haziness involving the left maxillary and ethmoidal sinuses with retained secretions and thickened mucosa. (Fig. No.4)

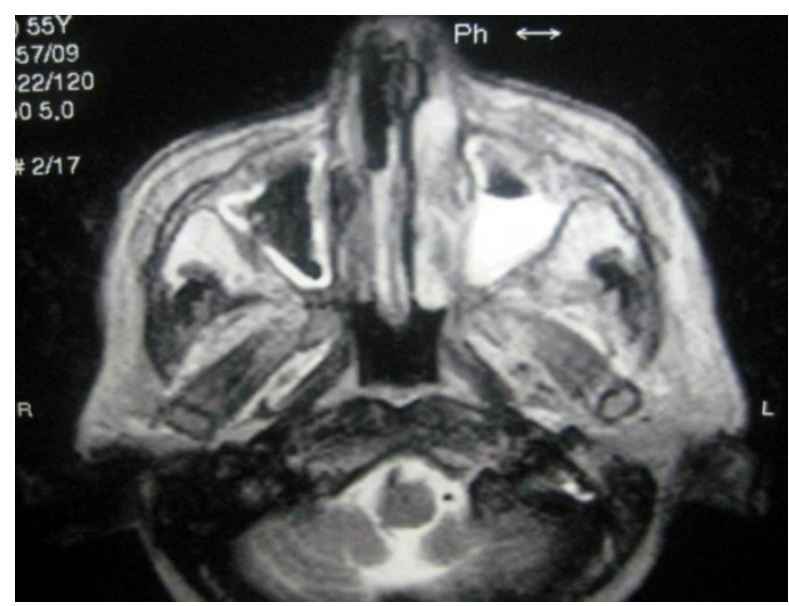

Figure 4. Showing haziness in left maxillary sinus
Diagnostic nasal endoscopy revealed mucopus in the middle meatus. No polyps or growth were seen in the nasal cavity. A swab taken from the palatal ulcer was suggestive of infection with rhizopus species. (Fig.no.5)

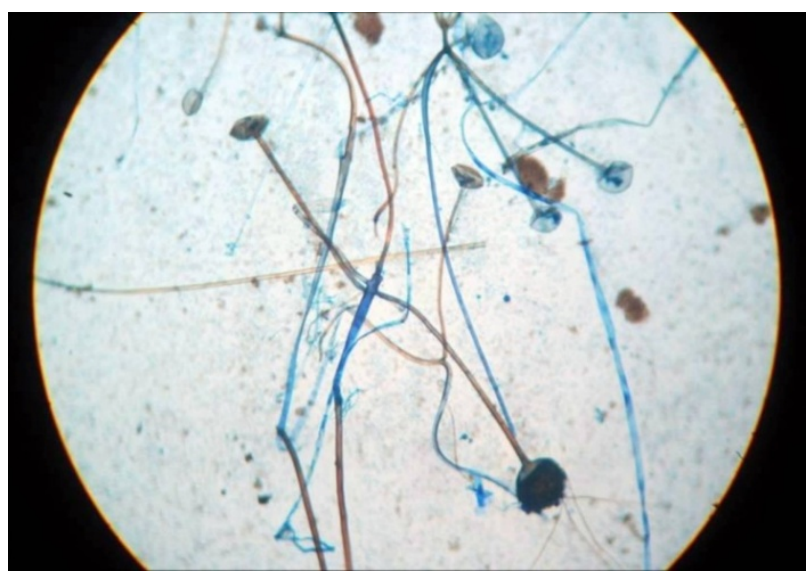

Figure 5. Showing rhizopus

Initial biopsy of the necrotic lesion over the hard palate was inconclusive showing only inflamed necrotic tissue. Patient underwent an endoscopic medial maxillectomy and debridement of the palatal necrotic tissue. The HPE of the inferior turbinate mucosa, palatal tissue and the antral mucosa were suggestive of zygomycosis. (Fig. No.6)

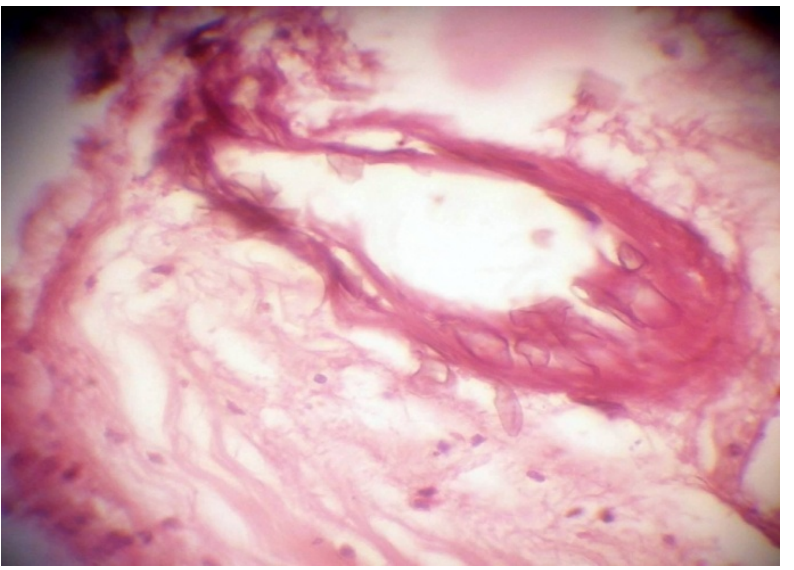

Figure 6. Showing vascular fungal invasion in HPE

Inj. Amphotericin B was started in the dose of 1 $\mathrm{mg} / \mathrm{kg} /$ day in $5 \%$ dextrose intravenously and the dose was gradually increased to $1.5 \mathrm{mg} / \mathrm{kg} /$ day and was given for a period of 4 weeks. The diabetes was kept under control with human mixtard insulin injections. The patient started showing significant improvement after 10 days of treatment and had full recovery of facial palsy and was able to walk without any unsteadiness at the end of amphotericin therapy. Patient has remained asymptomatic after 18 months of follow up.

\section{Discussion}

Mucormycosis is one of the most rapidly progressing and lethal form of fungal infection in humans which usually 
begins in the nose and paranasal sinuses. It is best known for its rhinocerebral presentation. ${ }^{3}$ Although first described by Paltauf in 1885, the term rhinocerebral mucormycosis was coined by Baker. ${ }^{5}$ About 70 percent of patients with this disease have been found to have diabetic ketoacidosis. ${ }^{6}$ Other predisposing factors are renal failure, long term corticosteroid therapy, desferrioxamine and immunosuppressive therapy, burns, organ transplant, lymphoma, leukemia and AIDS. ${ }^{1}$

Rhinocerebral mucor mycosis usually originates in the nasal cavity and paranasal sinuses, extends onto palate, pharynx and orbit. ${ }^{7}$ The progression of the disease is by direct spread or hematogenous dissemination facilitated by angioinvasion. Intracranial extension via perineural spread has also been documented. ${ }^{2}$ Muscles are usually spared. ${ }^{3}$ Angioinvasion by the hyphae produces a fibrin reaction and the development of mucor thrombi which occludes the arteries leading to ischaemia and infarction producing the characteristic black necrotic crusts. The infection spreads rapidly to adjacent sinuses, orbit and the cranium via the ethmoid bone and the orbital vessels. ${ }^{3}$

A classical presentation of the disease is a poorly controlled diabetic patient presenting with fever, facial pain and swelling, nasal congestion and eyelid swelling consistent with acute rhinosinusitis or periorbital cellulitis. ${ }^{1}$ They may also have retro orbital headache, occasional blood tinged nasal discharge, anaesthesia over the cheek, cranial polyneuropathy, proptosis and opthalmoplegia. Examination of the nasal cavity may reveal thick nasal discharge with black necrotic areas over the turbinates and septum. Though this finding is highly suggestive of mucormycosis, this occurs in only 40 percent of the cases. ${ }^{2}$

Orbital involvement in rhinocerebral mucormycosis occurs in 66-100 percent of cases resulting in chemosis, proptosis and opthalmoplegia. Blindness may result from central retinal artery occlusion or involvement of the optic nerve via direct orbital extension. ${ }^{2}$

Intracranial spread of the disease indicates extensive infection and grave prognosis due to encasement and thrombosis of major intracranial vasculature. Bilateral eye signs are suggestive of cavernous sinus thrombosis. ${ }^{2}$ Involvement of cerebellar artery is very rare. Intracranial extension without orbital involvement is also uncommon.

Early diagnosis and an immediate initiation of treatment of treatment are absolutely essential to ameliorate the poor prognosis. A diabetic patient presenting with features of rhinosinusitis with black necrotic slough in the nose and palate, a diagnosis of rhinocerebral mucor mycosis should be suspected. Initial CT imaging shows nonspecific mucosal thickening with soft tissue infiltration. ${ }^{8}$ Bone erosion in CT is strongly diagnostic; but, radiological findings often lag behind the clinical progression. MRI is more sensitive in detecting meningeal and intracranial vascular occlusion often before the patient develops the clinical signs. ${ }^{2}$ The diagnosis of mucormycosis is confirmed histologically by demonstrating angioinvasion by irregular, broad nonseptate hyphae that branch at right angles. The hyphae of other fungi are septate and branch at acute angles. Sterile culture doesn't rule out mucormycosis as organisms may be killed during preparation for tissue culture. ${ }^{2}$ However, in our case the diagnosis was by culture as the initial biopsy was inconclusive.

If mucormycosis is suspected, initial empirical therapy with antifungal drugs should begin while the diagnosis is being confirmed. Surgical treatment of the infected and necrotic tissue is the standard treatment along with the medical treatment. The angioinvasion and the tissue necrosis make it difficult for the chemotherapeutic agents to penetrate the tissue in which the fungus thrives. Combined treatment increases the survival to 78 percent as compared to 57.5 percent with medical treatment alone. In the presence of intracranial extension, surgical debridement may not be possible and almost all the cases are fatal. ${ }^{2}$

Several surgical procedures have been described ranging from debridement of the mucosa, Caldwell-Luc surgery, medial maxillectomy, ethmoidectomy, sphenoidectomy, radical maxillectomy with orbital exenteration. ${ }^{3}$ Both endoscopic and open approaches have been used. The standard medical therapy is Amphotericin B in a dose of 1-1.5 $\mathrm{mg} / \mathrm{kg} / \mathrm{day}$ for several weeks depending upon the clinical response and degree of nephrotoxicity ${ }^{3}$ Our patient received intravenous Amphotericin for 4 weeks after the debridement. Other modalities of treatment tried are hyperbaric oxygen therapy and nasally nebulzed Amphotericin $\mathrm{B},{ }^{3}$ oral Posaconazole $^{8}$

Reported survival rates range from 21 to 70 percent. $^{2}$ Proper management of diabetes adds to improved prognosis.

\section{Conclusions}

Rhinocerebral mucormycosis is an aggressive condition and can be life threatening in immunocompromised patients. Early diagnosis and combined modality of medical and surgical treatment is required for better prognosis.

\section{REFERENCES}

[1] Kyrmizakis DE, Doxas PG, Hajiioannou JK and Papadakis CE. Palate ulcer due to mucormycosis. J Laryngol Otol 2002; 116:146-47

[2] Munir N and Jones NS. Rhinocerebral mucormycosis with orbital and intracranial extension: A case report and review of optimum management. J Laryngol Otol 2006;121:192-95

[3] Yadav SPS and Goel AK. Rhino-orbital mucormycosis - A case report. International J of Pediatric Otolaryngology Extra 2010; 5:9-12

[4] Hosseini SMS and Borghei P. Rhinocerebral mucor mycosis: pathways of spread. Eur Arch Otorhinolaryngol 2005; 262:932-38 
[5] Baker RD. Mucor mycosis; a new disease? J Am Med Assoc. $1957 ; 163: 805-8$

[6] Spellberg B,Edwards J and Ibrahim A. Novel perspectives on mucormycosis: Pathophysiology, presentation and management. Clin Microbiol Rev 2005; 18:556-69

[7] Bansal S, Grover G,Grover M and Gupta AK. Isolated sphenoid mucormycosis presenting as visual impairment: changing trends? Am J of Otolaryngol Head Neck Surg 2010; 31:64-66

[8] Scheckenbach K, Cornely O, Hoffmann TK, Engers R, Bier H, Chaker A et al. Emerging therapeutic options in fulminant invasive rhinocerebral mucormycosis. Auris Nasus Larynx $2010 ; 37: 322-28$ 